Textures and Microstructures, Vol. 34, pp. 23-32 Reprints available directly from the publisher Photocopying permitted by license only
(C) 2000 OPA (Overseas Publishers Association) N.V.

Published by license under the Gordon and Breach Science Publishers imprint. Printed in Malaysia.

\title{
EFFECT OF CRYSTAL ROTATION ON SPRINGBACK OF INTEGRATED CIRCUIT LEADFRAMES
}

\author{
K.C. CHAN* and S.H. WANG \\ Department of Manufacturing Engineering, The Hong Kong Polytechnic \\ University, Yuk Choi Road, Hung Hom, Kowloon, Hong Kong
}

(Received 18 October 1999)

It is well known that crystal rotation which occurs in deformation processes may affect the mechanical and plastic properties of textured materials. In the present paper, the effect of crystal rotation on springback of a textured copper strip is examined. A model proposed previously by the authors for the prediction of springback of narrow and thin strips has been further extended to take crystal rotation into consideration. Based on the measured orientation distribution function of the strip, it is found that the influence of crystal rotation on the deformation behavior of the strip is significant. A reduction in elastic modulus, and circumferential and radial stresses are obtained. An improvement in the predicted springback angle is observed when crystal rotation is taken into consideration, though more research efforts still require to further enhance the accuracy of the model.

Keywords: Crystal rotation; Springback; Integrated circuit leadframe

\section{INTRODUCTION}

Recently, the authors have proposed an analytical model (Chan and Wang, 1999) based on a crystallographic-based yield criterion and the concept of relaxed constraints to describe the springback behavior of integrated circuit leadframes. In their model, two major sources of plastic anisotropy, namely, crystallographic textures and grain shape are considered. The trend of their prediction is found to be in consistence with experimental findings. It is well established that the change

\footnotetext{
* Corresponding author.
} 
of textures during cold deformation may affect the elastic and plastic properties of a material. Some published works have investigated the effect of crystal rotation on mechanical properties and formability of sheet metals (Chan and Lee, 1992; Chan, 1995; 1996). It has been reported that the evolution of yield locus of polycrystalline metals is related to the change of crystallographic textures (Chan and Lee, 1992). Chan has also illustrated that the effect of crystal rotation on limit strains of textured materials under equi-biaxial tension is significant. The incorporation of texture change in the analysis of sheet metal forming is considered to be essential. The effect of crystal rotation on springback of narrow strip has not yet been explored. In the present work, a model taking into consideration of crystal rotation will be further proposed to predict the springback behavior of narrow metal strips.

\section{MODELING}

In the paper, the model proposed by Chan and Wang (1999) will be modified to take into consideration of crystal rotation. According to their model, elements with infinitesimal dimensions in the radial and circumferential direction are assumed. Each element is assumed to have the same texture as the bulk sheet and is subjected to a plane stress state, and a crystallographic-based yield criterion is adopted in their paper. By satisfying the plane stress and the force equilibrium conditions, the stress and strain states of each element can be determined. For details of the model, it can be referred to the paper of Chan and Wang (1999). In the present model, three coordinate systems as shown in Fig. 1 are adopted. The sample coordinate system is used to describe the macroscopic behavior of a sheet. For each infinitesimal element, a reference coordinate system, i.e., rolling direction (RD), transverse direction (TD), normal direction (ND), is used to define the crystallographic texture. Since the crystallographic-based yield function is referred to the $\langle 100\rangle$ axes of the cube orientation, a cube coordinate system has to be used in determining the stress and strain of each element. The macroscopic behavior of the strip can then be determined by transforming the stress and strain of each element to the sample coordinate system.

In the model, the strip is considered to consist of $g$ groups of grains each with a certain volume fraction. Each element is assumed to have 


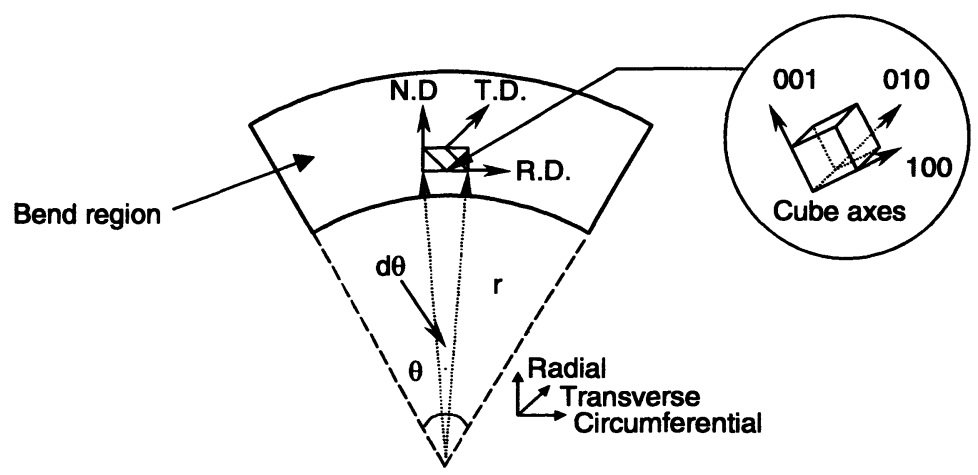

FIGURE 1 Geometry of a bent narrow strip and referential coordinate.

same texture as the bulk sheet, and have to satisfy the force equilibrium equation (Lubahn and Sachs, 1950)

$$
\begin{aligned}
& \left(\sigma_{r(s)}+\mathrm{d} \sigma_{r(s)}\right)(w+\mathrm{d} w)(r+\mathrm{d} r) \mathrm{d} \theta-\sigma_{r(s)} w r \mathrm{~d} \theta \\
& \quad-2 \sigma_{\theta(s)} \mathrm{d} r(w+\mathrm{d} w) \sin \mathrm{d} \theta / 2=0,
\end{aligned}
$$

where $\sigma_{r}, \sigma_{\theta}$ are the radial stress and circumferential stress, the subscript $s$ denotes the sample coordinate system, $r$ is the arbitrary radius, and $w$ is the width of the strip.

The crystallographic-based yield criterion adopted in this paper is of the following form (Montheillet et al., 1985):

$$
\begin{aligned}
F\left(S_{(c)(g)}\right)= & \alpha\left[\left|S_{11}-S_{22}\right|^{n}+\left|S_{22}-S_{33}\right|^{n}+\left|S_{33}-S_{11}\right|^{n}\right] \\
& +2 \beta\left[\left|S_{23}\right|^{n}+\left|S_{31}\right|^{n}+\left|S_{12}\right|^{n}\right]=\left(\sqrt{6} \tau_{c}\right)^{n}
\end{aligned}
$$

where $S_{i j}$ are the deviatoric stresses referring to the cube axes, the subscript $c$ denotes the cube coordinate system. $\alpha, \beta$ and $n$ are parameters of the yield function.

The stress tensor $S_{(c)}$ is related to the $\varepsilon_{(c)(g)}$ by applying the normality rule to the analytical yield function of Eq. (2) as follows:

$$
\boldsymbol{\varepsilon}_{(c)(g)}=\lambda \frac{\partial F\left(\boldsymbol{S}_{(c)(g)}\right)}{\partial \boldsymbol{S}_{(c)(g)}} .
$$


By varying the parameters in the strain tensor until the stress tensor for each element satisfies the plane stress and the force equilibrium condition as represented by Eq. (1), the stress for each element can be determined. After bending to a small angle increment, the change of orientation for each grain is considered. The amount of crystal rotation depends on the initial crystallographic orientation $\{h k l\}\langle u v w\rangle$ and the strain. The new orientation $T^{\prime}$ is related to the initial orientation $T$ by

$$
T^{\prime}=(I-\Omega) T,
$$

where

$$
\Omega_{c}=\left(\mathrm{d} w_{(c) i j}\right)=\frac{1}{2} \sum_{s=1}^{12}\left(a_{j}^{\mathrm{s}} b_{i}^{\mathrm{s}}-a_{i}^{\mathrm{s}} b_{j}^{\mathrm{s}}\right) \gamma^{\mathrm{s}} .
$$

The shear strain $\gamma$ is related to the strain increment of $\varepsilon_{(c) i j}$ by

$$
\left(\begin{array}{c}
\varepsilon_{22(c)(g)} \\
\varepsilon_{33(c)(g)} \\
2 \varepsilon_{23(c)(g)} \\
2 \varepsilon_{13(c)(g)} \\
2 \varepsilon_{12(c)(g)}
\end{array}\right)=E\left(\begin{array}{c}
\gamma_{1} \\
\gamma_{2} \\
\gamma_{3} \\
\gamma_{4} \\
\gamma_{5}
\end{array}\right),
$$

where $E$ is a square matrix that denotes the direction cosines of the slip systems with respect to the cube coordinate axes.

There are many ways of choosing 5 independent slip systems out of the 12 possible in cubic metals. The criterion for selecting the slip systems is based on the Bishop and Hill's principle of maximum work. However, ambiguities still exist in the selection of the active slip systems. For simplicity, Renouard and Wintenberger's method (1981) involving the minimization of the second-order term for the plastic work is chosen. According to their method, a small strain is applied to each possible set of the slip systems. This leads to a small crystal rotation. The second-order work done $\mathrm{d} W^{\prime}$ for each possible combinations can be determined by

$$
\mathrm{d} W^{\prime}=\sigma_{i j(c)(g)}^{\prime} \mathrm{d} \varepsilon_{i j(c)(g)}^{\prime},
$$


where $\sigma_{i j(c)(g)}^{\prime}$ and $\mathrm{d} \varepsilon_{i j(c)(g)}^{\prime}$ are the new stress and strain components respectively due to crystal rotation and strain hardening of the crystal. The set of slip systems that corresponds to the minimal second-order plastic work will then be selected.

After a new orientation $T^{\prime}$ has been determined from the above formulation, the metal strip is further deformed to a small angle increment and the strain tensor is re-calculated. The above procedures will be repeated until the final bending angle is reached.

For a specific bending radius and angle, when the stresses for all elements are determined, the bending moment per unit length can be obtained by integrating the circumferential stress $\sigma_{\theta}$ across the thickness and the width direction with respect to the current center of curvature:

$$
M_{\mathrm{B}}=\int \sigma_{\theta} w r \mathrm{~d} r .
$$

By assuming that the springback is resulted from fully elastic recovery, the curvatures of unstretched fiber before and after unload are related by the following expressions:

$$
\frac{1}{r_{\mathrm{u}}^{\prime}}=\frac{1}{r_{\mathrm{u}}}-\frac{M_{\mathrm{B}}}{E I}
$$

The unstretched fiber is taken as the springback axis so that the length of the fiber before and after springback is the same. Hence, the final angle after springback is expressed as

$$
\theta^{\prime}=\frac{r_{\mathbf{u}}}{r_{\mathbf{u}}^{\prime}} \cdot \theta
$$

It is worth to discuss that the anisotropic elastic properties of the material are also related to the crystallographic texture. The effective Young's modulus, $E_{d}$, along any direction $d$ indexed by Miller indices [hkl], can be given by

$$
\frac{1}{E_{d}}=s_{11}+\frac{\left(2 s_{12}-2 s_{11}+s_{44}\right)\left(k^{2} l^{2}+l^{2} h^{2}+h^{2} k^{2}\right)}{\left(h^{2}+k^{2}+l^{2}\right)^{2}},
$$


where $s_{11}, s_{12}$ and $s_{44}$ are the elastic compliance constants of a strip. In the present model, the change of elastic modulus will also be determined by Eq. (10) due to crystal rotation during the bending process.

\section{RESULTS AND DISCUSSION}

In this paper, the springback behavior of a narrow metal strip having $0.15 \mathrm{~mm}$ thickness and $0.20 \mathrm{~mm}$ width is examined. In the bending process, the die angle of $90^{\circ}$ and die radii of 1.0, 1.5, 2.0, 2.5 and $3.0 \mathrm{~mm}$ are used. The crystallographic textures of the copper alloy are measured by a Philips X-ray diffractometer and presented by $\{110\}\langle 112\rangle(67.13 \%)+\{102\}\langle 211\rangle(14.21 \%)+\{010\}\langle 001\rangle(10.19 \%)+$ $\{323\}\langle 131\rangle(8.47 \%)$. Figure 2 shows the measured ODF of the copper alloy. The random orientations are not taken into account in the simulation for simplifying the calculation. For zero scatter width, the parameters $\alpha, \beta, n$ in Eq. (2) are chosen to be $1.6,0.46$ and 0.51 respectively (Montheillet et al., 1985). The microscopic work hardening exponent for the copper alloy is assumed to be 0.59 . The elastic modulus of the strip along the rolling direction was measured to be $125 \mathrm{GPa}$.

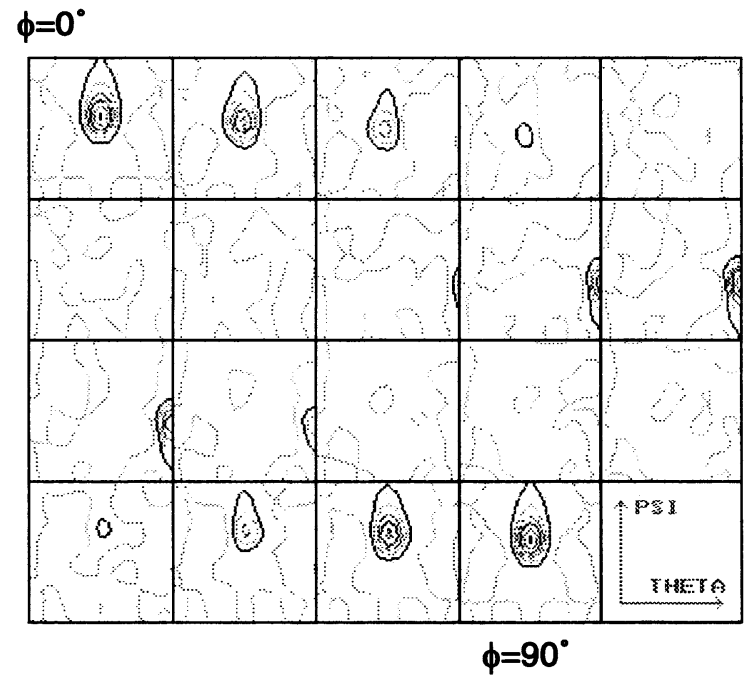

Level: 1-2-3-4-5-6-7-8-9-10-11-12-13-14

FIGURE 2 Orientation distribution function of the copper strip. 
Figures 3 and 4 show the predicted circumferential and radial stresses of the strips deformed along the RD and TD for the die radius of $1.0 \mathrm{~mm}$. For comparison purposes, the stresses obtained by the model without considering crystal rotation is also illustrated in the figures. It is clearly observed that smaller circumferential stresses and the radial stresses are predicted by the present model for both sheet directions.

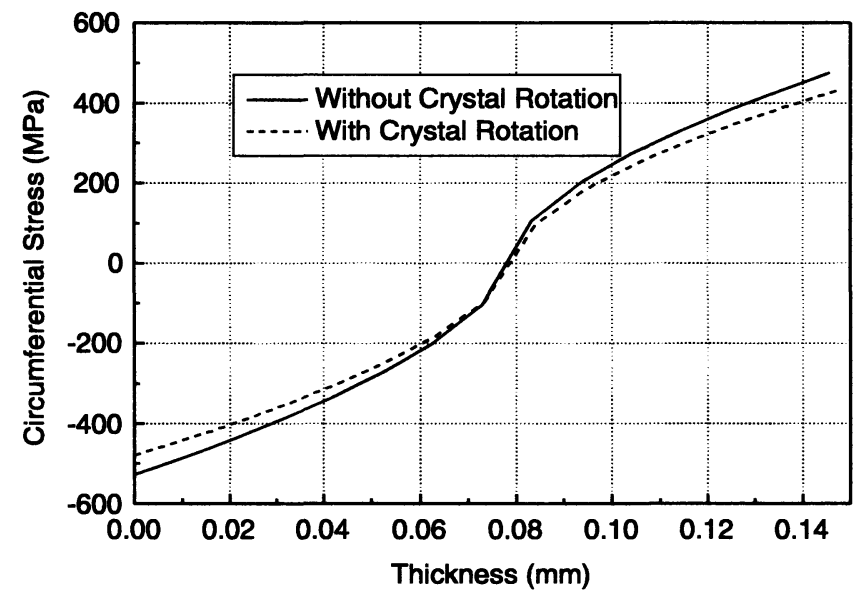

FIGURE 3 Predicted circumferential stresses.

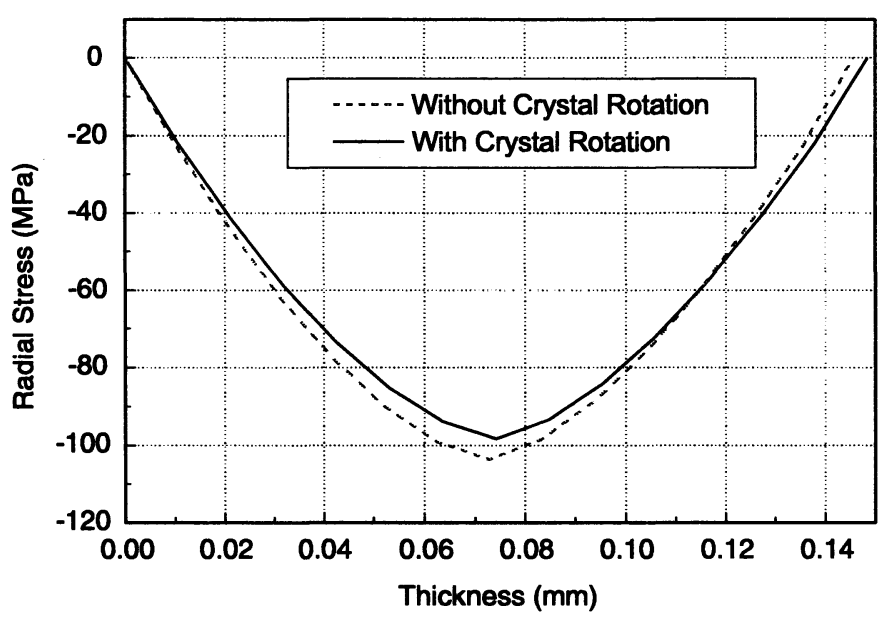

FIGURE 4 Predicted radial stresses. 
There seems to be a tendency for the grains to rotate to the new orientations which will result in smaller stresses. Figure 5 illustrates the change of elastic modulus along the thickness direction of the strip. A significant reduction in elastic modulus is found in the inner and outer regions of the strip for the die radius of $3.0 \mathrm{~mm}$, but only a slight reduction is observed for the die radius of $1.0 \mathrm{~mm}$. It is considered the change of elastic modulus is related to the amount of deformation experienced by the material, as a large deformation might lead to a greater crystal rotation.

The springback behavior of the strip predicted by the present model is compared with that predicted by the model without considering crystal rotation and the experimental findings, as shown in Fig. 6 . The general trend predicted by the present model is similar to that without crystal rotation. The springback angle increases with increasing die radius, and a larger springback angle is obtained for the strip deformed along the rolling direction. However, the discrepancy between the present predictions and the experimental findings is reduced. For large die radius (say, greater than $2.5 \mathrm{~mm}$ ), a smaller springback angle is predicted which is found to be in better agreement with the experimental results. Whereas for small die radius, a smaller spring back angle is predicted, which is more consistent to the experimental results. Although some improvements are observed, discrepancies between the theoretical

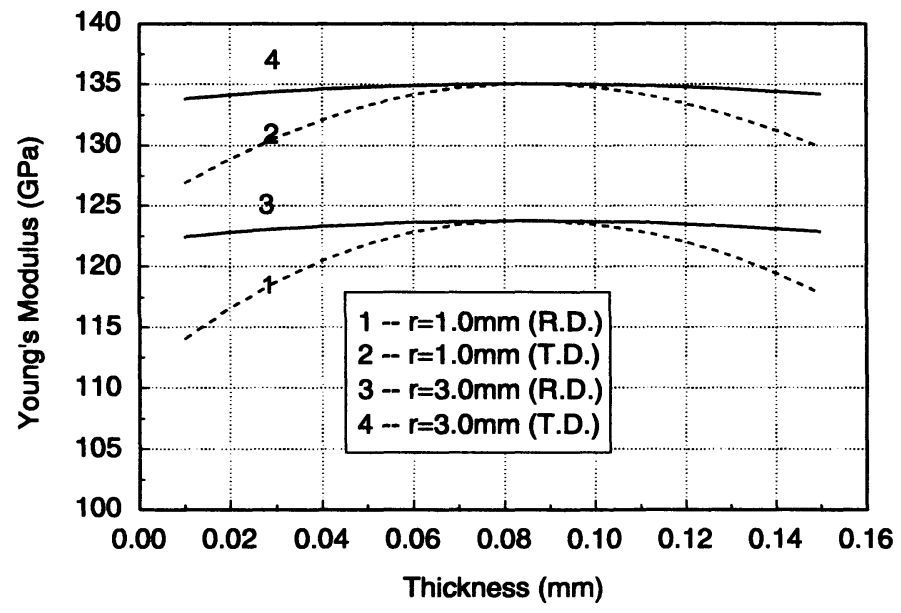

FIGURE 5 Changes of elastic modulus. 


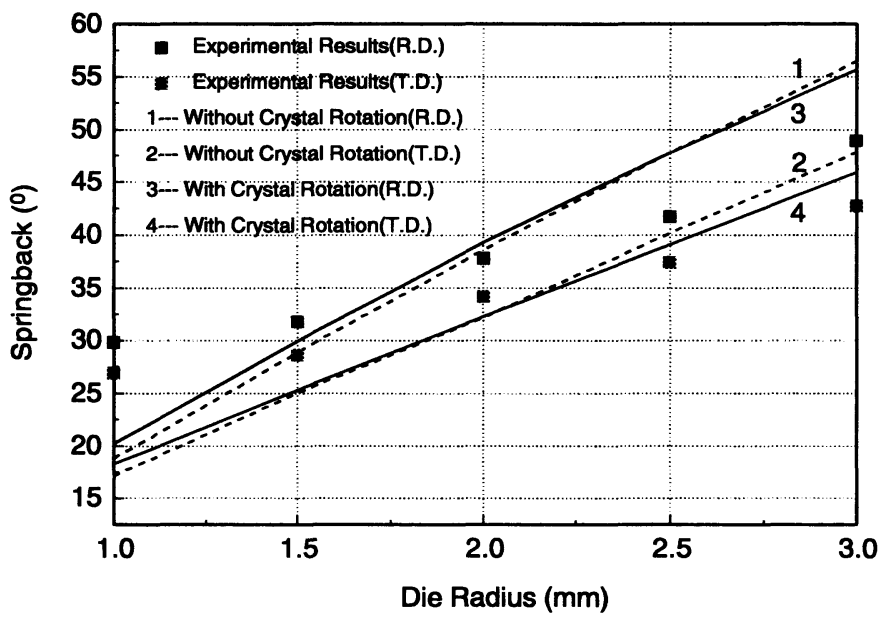

FIGURE 6 Predicted springback behavior.

predictions and the experimental results still exist. It is considered that the assumption of pure bending is too ideal for the roller bending process which is adopted in the experimental investigations. In order to improve the accuracy of the prediction, the incorporation of the present approach into a finite element formulation will be very worthwhile to consider.

\section{CONCLUSIONS}

In this paper, the effect of crystal rotation on springback of integrated circuit leadframes has been investigated. It is found that both the predicted circumferential and radial stresses of a bent copper strip are reduced with the consideration of grain rotation. It is also observed that there is a significant reduction in the elastic modulus when the deformation is large. The influence of crystal rotation on plastic and elastic properties of the strip is shown to be significant. The springback behavior of the strip predicted by the present model is compared with that without considering crystal rotation and the experimental results. The trend of the predictions is in better agreement with the experimental results. It is considered that in order to further improve the accuracy of 
the predictions, the present approach should be incorporated into a finite element formulation which can better describe the roller bending process.

\section{Acknowledgments}

The authors wish to express their gratitude for the financial support of the project rendered from the Hong Kong Research Grant Council and the support from the Hong Kong Polytechnic University.

\section{References}

Chan, K.C. (1995) Textures and Microstructures, 23, 257.

Chan, K.C. (1996) Textures and Microstructures, 26-27, 547.

Chan, K.C. and Lee, W.B. (1992) Proceedings of Advances in Engineering Plasticity and its Applications, Elsevier Science Publisher BV, p. 547.

Chan, K.C. and Wang, S.H. (1999) Materials Science and Engineering, A270, 323.

Lubahn, J.D. and Sachs, G. (1950) J. Eng. Ind., 72, 201.

Montheillet, F., Gilormini, P. and Jonas, J.J. (1985) Acta Metall., 33, 705.

Renouard, M. and Wintenberger, M. (1981) CR Acad. Sci. Paris, B292, 385. 